\title{
Risk Factors and Etiologies of Ischemic Stroke in Young Adults: A Hospital-based Study in Bangladesh
}

\author{
Habib R $\mathbf{R}^{\mathrm{a}}$, Hosen $\mathbf{I}^{\mathrm{b}}$, Islam RN', IslamR ${ }^{\mathrm{d}}$, Bhowmik NB
}

\begin{abstract}
Background: Stroke is an important cause of disability among adults and is one of the leading causes of death worldwide. Strokes in young adults can have a significant impact on the affected individuals, their families and societies in general as the affected patients are in the economically productive period of their lives. This study was aimed to find out the demographic profile and explore the associated risk factors of the first-ever ischemic stroke of young adults.
\end{abstract}

Methods: This retrospective study reviewed the records of 64 patients, age 18-49 years, ischemic stroke patients consecutively admitted in the Neurology department, BIRDEM General Hospital during the period January 2016 to June 2017. Study variables included hematological and biochemistry investigations and radiological and imaging findings. Stroke sub-typing was done following to TOAST criteria. Data of follow-up data at 3 month were also included. Data were expressed as number (percent) and managed by SPSS for Windows Version 15.

Results: Of the 634 patients diagnosed as acute ischemic stroke 64 (9.90\%) were in the 18-49 (yr) age range and the majority (55.4\%) were male. Stroke was more common (47 out of 54) among the 31-49 years category as compare with the $<30$ years category. Stroke patients presented with diabetes mellitus(87.03\%), arterial hypertension (44.4\%), dyslipidemia (23.5\%) and cigarette smoking (31.48\%). Family history of stroke was present in $33.5 \%$ cases. Ten (6.51\%) patients showed hypercoagualable state.Small vessel occlusion (SVO) found in $48.40 \%$ cases followed by large artery atherosclerosis (21.86\%).Patients with SVO stroke presented with lacunar syndromes and lesions in Basal ganglia(12.1\%), Thalamus(6.6\%), Subcortical region (0.9\%), Internal capsule (20.0\%) and brain stem(11.3\%). Thrombolysis was not done in any of patients. Most of the patients were on an anti-platelet treatment and majority (85.4\%) receiving aspirin alone. Strokes left $48(75.5 \%)$ patients with moderate to moderately severe disability on discharge from the hospital.Mortality was lower (6.3\%) and had good recovery 35 (54.6\%).

Conclusion: Ischemic stroke in the young adults accounted for $9.90 \%$ of the hospital admission with stroke. Those suffered from ischemic stroke had presence of modifiable risk factors. Predominance of SVO among them not only indicated stroke etiology but also predicted good short term prognosis.

Keywords: Young adult, Ischemic stroke, TOAST classification study, National Institutes of Health Stroke Scale (NIHSS),Modified Rankin Scale (mRS).

(BIRDEM Med J 2018; 8(2): 138-144)

\section{Author Information}

a. Dr. Rumana Habib, Assistant Professor, Department of Neurology, BIRDEM General Hospital, Dhaka

b. Dr. Ikram Hosen, Assistant Registrar, Department of Neurology, BIRDEM General Hospital.

c. Dr. Rashedul Islam, Registrar, Department of Neurology, BIRDEM General Hospital, Dhaka

d. Dr. Nirmalendu Bikash Bhowmik, Professor, Department of Neurology, BIRDEM General Hospital, Dhaka

Address of correspondence: Dr. Rumana Habib, Assistant Professor, Department of neurology, BIRDEM General Hospital, Dhaka. Email:drhrumana4@gmail.com

Received: December 14, 2017 Accepted: February 28, 2018

\section{Introduction}

Ischemic strokes in young adults can have a significant impact on the affected individuals, their families, and societies in general, as the patients are affected in the economically productive period of their lives. Almost two thirds of the global burden of stroke is borne by those in developing countries. ${ }^{1}$ The etiologies of ischemic strokes in young adults are different and more diverse as compared to those observed in the elderly. It is important to identify the causative factors in young stroke patients in order to prevent recurrences appropriately. ${ }^{2}$ 
The etiology of ischemic stroke affects prognosis and outcome. ${ }^{3}$ Clinical practice guidelines recommend different treatments according to stroke mechanism. Consequently, timely and accurate diagnosis of stroke mechanism is required. Supplementation of clinical features with information on infarction patterns and vascular territories obtained from initial brain images may enable individualization of the diagnostic workup and diagnosis of stroke mechanism. A system for categorization of subtypes of ischemic stroke mainly based on etiology has been developed for the Trial of Org 10172 in Acute Stroke Treatment (TOAST). ${ }^{4}$

The current study was conducted to identify ischemic stroke risk factors and etiological subtypes according to the TOAST criteria in young patients admitted under Neurology department of a tertiary care hospital in Dhaka, Bangladesh.

\section{Methods}

We conducted a retrospective review of 64 consecutive patients aged 18-49 yearswhowere admitted under the Department of Neurologyof tertiary care hospital, from January 2016 to June 2017 with ischemic stroke.Eligibility for the study was in accordance with the World Health Organization (WHO) definition of stroke. 5

Ischemic stroke was defined as a sudden focal neurologic deficit with imaging-confirmed cerebral infarction. We excluded cases of intracranial hemorrhage, subarachnoid hemorrhage, and cerebral venous thrombosis.

In history taking, patients were enquired specifically about previous deep vein or pulmonary thrombosis, arthralgia, skin rashes, miscarriages, a family history of thrombosis or drug abuse. The clinical examination focused on the nervous and cardiovascular systems. The presence and equality of all peripheral pulses, carotid and subclavianbruits, the cutaneous stigmata of hyperlipidaemias were sought. Other features such as a Homer's syndrome, Marfanoid habitus, skin laxity and joint hypermobility, livedoreticularis, vasculitic rash, splinter haemorrhages, oral and genital ulcers and venepuncture marks were not be overlooked.

As a matter of protocol, all these stroke patients admitted under neurology department underwent routine hematological and biochemistry tests (complete blood count, serum creatinine, blood glucose and lipid profile), chest X-rays, electrocardiography, and brain imaging including computed tomography (CT) and/or magnetic resonance imaging (MRI) at admission. Transthoracic echocardiography and Holter monitoring were performed in selected patients. More specific diagnostic testing (e.g. vasculitis profile) was performed when deemed necessary by the treating neurologist.

A structured questionnaire as appended was used to collect information on demographic variables, vascular risk factors, and stroke workup, and stroke subtype using TOAST criteria. Patients were labeled as hypertensive if systolic blood pressure was greater than $140 \mathrm{mmHg}$ or/and diastolic blood pressure was greater than $90 \mathrm{mmHg}$ during repeated measurements during the patient management in the hospital or if the patient was on antihypertensive drugs at the time of admission. Weclassified patient as diabetic if self reported fasting glucose level of the patient was $7 \mathrm{mml} / 1$ or more or if the patient was on hypoglycemic agents or insulin. Patients having serumhigh density lipid of $100 \mathrm{mg} / \mathrm{dL}$ or less and/or serum low density lipid of $100 \mathrm{mg} / \mathrm{dL}$ or more and/or fasting serum cholesterol of $200 \mathrm{mg} / \mathrm{dL}$ or more werelabeled as having dyslipidemia. Smokers were the patients who had smoked ten or more cigarettes for ten ormoreyears. Patients or their next of kin were briefed about the purpose and nature of the study. Written consent was obtained from the patients or their next of kin in case of incapacitation of the patients.

In our study National Institutes of Health Stroke Scale (NIHSS) score was documented for all young stroke patients in 24 hours of admission to establish the baseline stroke severity and identify individuals at greatest risk for hemorrhagic complications. The NIHSS score isa quantitative measure of stroke-related neurological deficitthat spans key aspects of the neurological examination: level of consciousness, language function, neglect, visual fields, eye movements, facial symmetry, motor strength, sensation, and coordination. Patients were prospectively followed up clinically and outcome of the enrolled patients were measured by in-hospital and 30 days mortality and Modified Rankin Scale (mRS) at discharge from hospital and at 30 day follow up at Neurology OPD. A modified Rankin Scale score of 0-2 at 30 days was defined as a favorable functional outcome. 


\section{Results}

Of a total number of 634 subjects with first ever stroke admitted to our institute from January 2016 to June 2017, 64 consecutive patients aged 18-49 years were recruited in our study $55.4 \%$ of the patients were male and the mean patient age was $38.9 \pm 7.1$ years.Age andgender distribution of study populationis shown in Figure 1 .

Identified risk factors for ischemic stroke were diabetes mellitus (87.03\%), hypertension (44.4\%), dyslipidemia $(23.5 \%)$ and cigarette smoking (31.48\%) out of whom twelve (22.2\%) were current smokers and seven (12.94\%) were former smokers. These are presented in Table I. Risk factors according to gender of study population are shown in Table II and overall glycemic status of the diabetic population is depicted in Table III.

\begin{tabular}{lcc}
$\begin{array}{l}\text { Table I. Risk factors and demographic characteristics } \\
\text { of study population (N=64) }\end{array}$ & \multicolumn{1}{c}{$\%$} \\
\hline Risk factor & 55 & 87.03 \\
\hline Diabetes & 29 & 46.29 \\
Hypertension & & 73.22 \\
Dyslipidemia & 23 & \\
High LDL cholesterol & 20 & \\
Low HDL cholesterol & 09 & \\
No dyslipidaemia & 13 & 20.31 \\
Cardiovascular disease & 06 & 12.96 \\
Coronary artery disease with & & \\
LV dysfunction & 02 & 5.55 \\
Valvular heart disease & 02 & \\
Atrial fibrillation & 0 & \\
Cardiomyopathy & 02 & \\
LV / LA thrombus & 01 & \\
LV apical myxoma & 14 & 21.86 \\
Carotid stenosis $>70 \%$ & & \\
Tobacco consumption & 14 & 22.22 \\
Current smoker & 05 & 9.25 \\
Past smoker & 04 & 3.703 \\
Chewing tobacco & 01 & 1.85 \\
High-risk alcohol consumption & 21 & 33.33 \\
Family history of stroke & 0 & 0 \\
Previous drug history esp. \\
anticoagulants
\end{tabular}

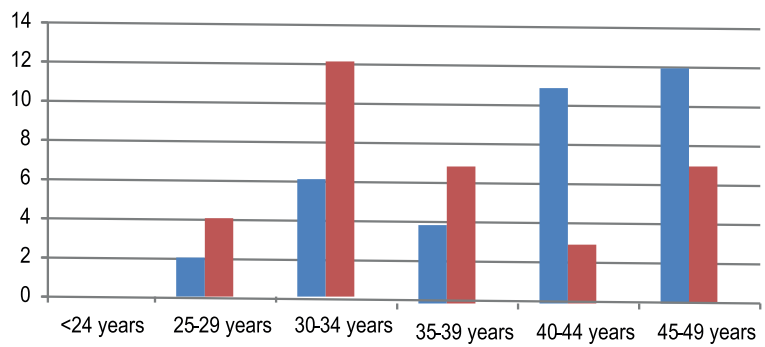

Figure 1. Age and gender distribution of study population $(N=64)$.

Table II. Risk factors according to genderofstudy population $(\mathrm{N}=64)$

Variable Male Female Total

Diabetes

Hypertension

(n)

(n)

(n)

Dyslipidemia

30

25

55

Coronary artery

LV dysfunction

$\begin{array}{llll}\text { Valvular heart disease with } \mathrm{AF} & 0 & 2 & 02\end{array}$

$\begin{array}{llll}\text { Nonvalvular Atrial fibrillation } & 0 & 2 & 02\end{array}$

/ flutter

Cardiomyopathy

LV / LA thrombus

$18 \quad 11 \quad 29$

LV apical myxoma

$26 \quad 20 \quad 46$

Carotid stenosis $>70 \%$

Current smoker

Past smoker

High-risk alcohol consumption 1

Family history of stroke

0

$2-0$

$2-02$

$\begin{array}{lll}1 & 0 & 01\end{array}$

$\begin{array}{lll}5 & 9 & 14\end{array}$

$14 \quad 0 \quad 14$

$5 \quad 0 \quad 5$

$\begin{array}{lll}1 & 0 & 1\end{array}$

\begin{tabular}{lll}
15 & 6 & 21 \\
\hline
\end{tabular}

Table III. Glycemic control of the diabetic patients (N=55)

\begin{tabular}{lcc}
\hline HbA1c & Number & Percent \\
\hline $7-9 \%$ & 12 & 21.81 \\
$9-11 \%$ & 26 & 48.05 \\
$>11 \%$ & 17 & 30.07 \\
\hline
\end{tabular}

All patients underwent $\mathrm{CT}$ scans and $38.4 \%$ underwent magnetic resonance imaging (MRI). Additionally, a carotid artery duplex scan was conducted in $95.7 \%$ of the patients, transthoracic echocardiography in $96.3 \%$, and transesophageal echocardiography in $2.3 \%$. A detailed work-up for coagulation disorders was conducted in $41(72 \%)$ of the patients.

The etiology of ischemic stroke affects prognosis, outcome, and management. The main categorizations of etiologic subtypes of ischaemic stroke in young adults in our study according to TOAST is shown in Table IV. 
Table IV. Etiological classification of according to gender ofstudy population $(\mathrm{N}=64)$

\begin{tabular}{lcccc}
\hline $\begin{array}{l}\text { TOAST classification of ischaemic } \\
\text { stroke }\end{array}$ & $\begin{array}{c}\text { Total } \\
(\mathrm{n}=64)\end{array}$ & $\begin{array}{c}\text { Percent } \\
(\%)\end{array}$ & $\begin{array}{c}\text { Male } \\
(\mathrm{n}=30)\end{array}$ & $\begin{array}{c}\text { Female } \\
(\mathrm{n}=24)\end{array}$ \\
\hline 1) large-artery atherosclerosis & 14 & 21.86 & 10 & 4 \\
2) small-vessel occlusion & 31 & 48.40 & 18 & 13 \\
3) cardioembolism & 13 & 20.31 & 9 & 4 \\
4) stroke of other determined etiology & - & - & & \\
5) stroke of undetermined etiology. & 6 & 6.2 & 3 & 3 \\
\hline
\end{tabular}

The patients with small vessel occlusive stroke presented with lacunar syndromes and lesions in Basal ganglia (12.1\%), Thalamus (6.6\%), Subcortical region $(0.9 \%)$, Internal capsule (20.0\%)and brain stem(11.3\%).Patients with stroke due to large artey atherosclerosis had signs of lesion in cortex such as aphashia, apraxia,neglect or cerebellar signs, compatible brain image findings and colordulpex showing $>50 \%$ stenosis of symotomaticextra -cranial artery. MCA territory infarct was found in $77.77 \%$ of patients. Again MCA territory infarct was commonest among patients suffering from Cardioembolic stroke.

The most common cause of cardioembolic stroke in our study was recent MI with severe LV dysfunction (7 patients, $12.96 \%$ ). Mitral stenosis and AF (5.55\%) with early cerebrovascular complications due to cardiogenic thromboembolism mechanisms. Also, other disturbances of cardiac rhythm(atrial flutter)were present in 2 of our patients. 2 cases of LV thrombus and a case of LA myxoma was found during our study period which were considered high risk source of cardioembolism. 6 of our patients were catagorized as of Undetermined group as two or more cause could be identified intheir cases as shown in Table V.

Table V. Undetermined class in study group

\begin{tabular}{lc}
\hline Etiology & Frequency (n) \\
\hline Hyperhomocysteinemia & 6 \\
Protein C deficiency & 1 \\
Systemic lupus erythematosus & 3 \\
(Positive for ANA, Antids DNA & \\
and Antiphospholipid antibody ) & \\
\hline
\end{tabular}

The carotid territory stroke were more frequentcompared to vertebrobasilar territory stroke $(84.2 \%$ verses $11.1 \%$ ), and strokes involving both territories $(4.7 \%)$.

Regarding treatment, thrombolysis was not performed in any of patients. Most of the patients were on an anti-platelet treatment with a majority $(85.4 \%)$ receiving aspirin alone. They were also managed with physical therapy from the beginning of the acute stage. To quantify the impairment caused by stroke NIHSS was done on admission and is shown in Table VI.

Table VI. Stroke severity of study population ( $\mathrm{N}=64)$ by NIHSS

\begin{tabular}{llc}
\hline Score & Stroke severity & $n$ \\
\hline 0 & No stroke symptoms & 0 \\
$1-4$ & Minor stroke & 14 \\
$5-15$ & Moderate stroke & 32 \\
$16-20$ & Moderate to severe stroke & 12 \\
$21-42$ & Severe stroke & 6 \\
\hline
\end{tabular}

Mean NIHSS score was 8.At the time of hospital discharge, $48(75.5 \%)$ patients with moderate to moderately severe disability with mRS score of 3 to 5.Status of patients on modified Ranking Scale score on discharge and on 30 day follow-upis shown in Table VII and Table VIII respectively. 
Table VII. Status of study population $(\mathrm{N}=64)$ by modified ranking scale on discharge

\begin{tabular}{llll}
\hline S1 No & No symptoms & $n$ & Percent \\
\hline 1 & $\begin{array}{l}\text { No significant disability. Able to carry out all usual activities, despite } \\
\text { some symptoms }\end{array}$ & 4 \\
2 & $\begin{array}{l}\text { Slight disability. Able to look after own affairs without assistance, } \\
\text { but unable to carry out all previous activities. }\end{array}$ & 9 \\
3 & $\begin{array}{l}\text { Moderate disability. Requires some help, but able to walk unassisted } \\
4\end{array}$ & $\begin{array}{l}\text { Moderately severe disability. Unable to attend to own bodily needs without } \\
\text { assistance, and unable to walk unassisted. }\end{array}$ & 24 \\
5 & $\begin{array}{l}\text { Severe disability. Requires constant nursing care and attention, bedridden, } \\
\text { incontinent }\end{array}$ & 12 \\
6 & Dead & 4 \\
\hline
\end{tabular}

Table VIII. mRS of enrolled patients $(\mathrm{n}=50)$ at 30 day follow up

\begin{tabular}{lll}
\hline S1 No & No symptoms & $n$ \\
\hline 1 & $\begin{array}{l}\text { No significant disability. Able to carry out all usual activities, despite } \\
\text { some symptoms }\end{array}$ & 2 \\
2 & $\begin{array}{l}\text { Slight disability. Able to look after own affairs without assistance, } \\
\text { but unable to carry out all previous activities. }\end{array}$ & 14 \\
3 & $\begin{array}{l}\text { Moderate disability. Requires some help, but able to walk unassisted } \\
4\end{array}$ & $\begin{array}{l}\text { Moderately severe disability. Unable to attend to own bodily needs without } \\
\text { assistance, and unable to walk unassisted. }\end{array}$ \\
5 & $\begin{array}{l}\text { Severe disability. Requires constant nursing care and attention, bedridden, } \\
\text { incontinent }\end{array}$ & 4 \\
6 & Dead & 4 \\
\hline
\end{tabular}

Mortality was lower $(4,6.3 \%)$ than disability and $(16,32 \%)$ had favorable functional outcome.

\section{Discussion}

By 2050 , it is anticipated that $80 \%$ of stroke patients will be from the developing regions of the world. ${ }^{5}$ Additionally, there is growing evidence for an increasing trend in the incidence of stroke in young adults. ${ }^{6}$ Accurate classification of ischemic stroke is critical for guiding treatment decisions and determining the prognosis of individual patients.

A slightly a higher male predominance was observed in the current study. Studies performed on ischemic stroke among the 15-45 years age group from India also reported a male preponderance. ${ }^{7}$ Similar findings have been reported from Denmark in cases of thromboembolic stroke. ${ }^{8}$ The proportion of cases was higher in the 31-45 years age group, which was similar to the findings reported by Nayaket al. ${ }^{2}$
Similar to the previously published results, the current study identified a relatively high frequency of modifiable risk factors for ischemic stroke. Of this diabetes, hypertension and dyslipidemia were the most common. Diabetes mellitus, a known risk factor of stroke, was found in 87.03 percent of our studied patients. At the same time uncontrolled Diabetes ( $\mathrm{HbA} 1 \mathrm{c}>9 \%$ ) was found to be a major contributor to risk in diabetic population. Diabetic patients were included in such large number in our study as they were recruited from, a tertiary care hospital run by the Diabetic Association of Bangladesh, only which, however, is 650-bed multidisciplinary hospital. Diabetes mellitus was also reported to be a risk factor for ischemic stroke from India $^{7}$ and Switzerland. ${ }^{9}$ Diabetes was not found to be a risk factor for ischemic stroke in Sweden ${ }^{10}$ and Taiwan. ${ }^{11}$ 
Essential hypertension was the most frequent in our study population, and renal and thyreotoxical hypertensions were rare. The atherogenic level of low density and high density lipoproteins (LDL/HDL) was present in 23.22 percent of young adult patients. Hypercholesterolemia and hypertriglyceridemia are known to be associated with stroke in young adultsLipskaet $a l^{7}$ did not find such an association in south Indian patients.

On the other hand, nicotinism seemed to be a significant risk factor in our young male population. We found no abuse of ephedrine ${ }^{12}$ or cocaine ${ }^{13}$ as risk factors of stroke in our group, although it was described in literature. A significant number of patients had a history of prior stroke and TIA. This observation emphasizes the need for aggressive primary and secondary prevention targeting the traditional modifiable risk factors for our young population.

The mechanism of stroke is an important characteristic of ischemic strokes and help predict outcomes after stroke and assess risk of stroke recurrence. Based on prior clinical trials, a subclassification scheme of five etioloiges of ischemic stroke has become widely accepted. They are(1) large artery atherosclerosis (LAA), (2) small vessel occlusion (SVO), (3) cardioembolism (CE), (4) stroke of other determined etiology (ODE) and (5) stroke of undetermined etiology. 4

The most common stroke subtype in the current study was small vessel occlussive (48.40\%), and was observed at a higher rate than that reported in previous studies of ischemic stroke in young adults $(24 \%-36 \%) .{ }^{14,15}$. Despite the high prevalence of the traditional risk factors for stroke in our cohort, only $21.66 \%$ of patients were found to have large vessel athereosclerosis. This could be due to the fact that only a few of our patients underwent extracranial/intracranialangiography and as aresult carotid and vertebral dissection, fibromuscular dysplasia, radiotherapy, homocystinuria, Moya-moya disease, Takayasu's arteritis remained undetected. Eitiology was undetrmined in $6.2 \%$ of cases . The most frequent reason for categorizing these patients as having an undetermined etiology was as two or more causes were identified which could be held responsible and in a few cases incomplete evaluation. Because patients did not have insurance and were therefore unable to afford the costs of radiological and biochemical diagnostic tests.

Observations from the first ever stroke registry of ischaemic stroke patients conducted in BIRDEM on patients aged between 30 and 90 years showed small vessel strokes were the most common accounting for $45.4 \%$ of all the patients followed by large vessel stroke in $32.5 \%$ of the cases. Cardioembolic stroke was present in $4.9 \%$ while etiology was not defined in $17.2 \%$ of the stroke cases. ${ }^{16}$

In a case controlled study of 214 first occurrence ischemic strokes in young adults from South India, Lipskaet $\mathrm{al}^{7}$ reported that cardioembolic stroke (25.2\%) was the most common stroke subtype, followed by large artery atherosclerosis (12.6\%). They reported that $11.2 \%$ of patients had ODE. In another retrospective study of 177 young adult ischemic stroke patients from South India, the most common etiologic subtype was atherothrombotic stroke $(24 \%)$, followed by cardioembolic stroke $(17 \%){ }^{2}$

In comparison, in a large European cross-sectional study of 3331 young adults with first-time ischemic stroke, $17 \%$ had cardioembolic disease, $13 \%$ had cervical artery dissection, $12 \%$ had small vessel occlusion, $9 \%$ had large-artery atherosclerosis, and $9 \%$ had other identified causes (not dissection), followed by thrombophilias, antiphospholipid antibody syndrome, systemic vasculitis, migraine with aura, and others. ${ }^{17}$

In the current study, cardioembolic stroke accounted for $20.31 \%$ of patients. The most common cause of cardioembolic stroke in the current study was ischamic heart disease. Previous studies from India have reported rheumatic heart disease and prosthetic valves contributing to almost two thirds of allcardioembolic strokes in young adults. ${ }^{2,7}$. This is in contrast to the results of studies from developed countries where etiologies such as patent foramen ovale (PFO) or atrial septal defects (ASD) were more common than valvular heart disease as a cause of stroke. ${ }^{15,16}$ Another reason for underestimation of PFO or ASD could be the very low rate of transesophageal echocardiography performance $(2.3 \%)$ in the current study.

The vascular territories and topographic localization of ischemic lesions were determined with reference to the anatomic maps of the dominant arterial territories proposed by Tatu et al. ${ }^{18}$ The carotid territory included 
the middle cerebral artery (MCA) territory, the anterior cerebral artery (ACA) territory, the internal carotid artery (ICA) territory, and the border zone territory. Ischemic lesions in posterior cerebral artery, basilar artery, vertebral artery, superior cerebellar artery, anterior inferior cerebellar artery, and posterior inferior cerebellar artery (PICA) were categorized as occurring in vertebrobasilar territory.

The Modified Rankin Scale (mRS) assesses disability in patients who have suffered a stroke and is compared over time to check for recovery and degree of continued disability. In a prospective study of 198 young ischemic stroke patients, Nedeltchev and colleagues reported that $68 \%$ of patients achieved an mRS score of $0-1,26 \%$ had an mRS score of $2-5$, and $3 \%$ had died within 3 months. ${ }^{19}$ Incontrast to these results, good functional outcomes at 30 day follow up ( $\mathrm{mRS}$ range $=0-2$ ) were observed in around $32 \%$ of patients in our cohort. These results suggest need for prompt diagnosis and early management for better outcome in young stroke patients in Bangladesh.

There are some limitations in the study. Apart from its retrospective nature, inadequate numbers, not all the patients underwent all the investigations, thereby making analysis and interpretations difficult. Being a tertiary care center, the referred patients' profiles may not be representative, creating a bias. Because of paucity of information, this study gives an idea of the sample size required to undertake more detailed studies with bigger sample sizes to explore the associations and risk factors. There were missing data on some of the variables. We were unable to follow up all the patients afterdischarge.Outcome variables such as mRS was not assessed at 30 day follow up.Life style factors such as physical inactivity, obesity,sleeping for $<6$ hours, life time history of migraine and high risk alcohol consumption,illicit drug abuse were not adequately stressed in our study.

Conflicts of interest: Nothing to declare.

\section{References}

1. Truelsen T, Bonita R, Jamrozik K. Surveillance of stroke: A global perspective. Int J Epidemiol2001;30:S11-16.

2. Nayak SD, Nair M, Radhakrishnan K, Sarma PS. Ischemic stroke in the young adult: Clinical features, risk factors and outcome. Natl Med J India1997;10:107-12.

3. Furie KL, Kasner SE, Adams RJ, Albers GW, Bush RL, Fagan $\mathrm{SC}$ et al .Guidelines for the prevention of stroke in patients with stroke or transient ischemic attack: a guideline for healthcare professionals from the american heart association/ american stroke association.Stroke. 2011 Jan;42(1):227-76.
4. Adams HP Jr ${ }^{1}$, Bendixen BH, Kappelle LJ, Biller J, Love $\mathrm{BB}$, Gordon DL, Marsh EE 3rd. Classification of subtype of acute ischemic stroke. Definitions for use in a multicenter clinical trial. TOAST. Trial of Org 10172 in Acute Stroke Treatment. Stroke 1993 Jan;24(1):35-41

5. Aho K, Harmsen P, Hatano S,Marquardsen J, Smirnov VE, Strasser T. Cerebrovascular disease in the community: results of a WHO collaborative study. Bull World Health Organ 1980;58:113-30

6. Mehindiratta MM, Agarwal P, Sen K, Sharma B. Stroke in young adults: A study from a university hospital in north India. Med Sci Monit 2004;10:CR535-41.

7. Hatano S. Experience from a mulitcentre stroke register: A preliminary report. Bull WHO 1976;54:541-43.

8. Lipska K, Sylaja PN, Sarma PS, Thankappan KR, Kutty VR, Vasan RS, et al. Risk factors for acute ischaemic stroke in young adults in South India. J Neurol Neurosurg Psychiatry 2007;78:959-63.

9. Lidegard O, Soe M, Andersen NM. Cerebral thromboembolism among young women and men from Denmark 1977-1982. Stroke 1986;17:670-75.

10. Arnold M, Halpern M, Meier N, Fischer U, Haefeli T, Kappeler $\mathrm{L}$, et al. Age dependent differences in demographics, risk factors, co-morbidity, etiology, management and clinical outcome of acute ischeamic stroke J Neurol2008;255:1503-07.

11. Kristensen B, Malm J, Carlberg B, Stegmayr B, Backman C, Fagerlund M, et al. Epidemiology and etiology of ischemic stroke in young adults aged 18 to 44 years in northern Sweden. Stroke 1997;28:1702-09.

12. Lee TH, Hsu WC, Chen CJ, Chen ST. Etiologic study of young ischemic stroke in Taiwan. Stroke 2002;33:1950-55.

13. Varona JF, Guerra JM, Bermejo F, Molina JA, Gomez de la Camara A. Causes of ischemic stroke in young adults, and evolution of the etiological diagnosis over the long term. Eur Neurol 2007;57:212-18.

14. Lisovoski F, Rousseaux P. Cerebral infarction in young people: A study of 148 patients with cerebral angiography. J Neurol Neurosurg Psychiatry 1991;54:576-77.

15. Bogousslavsky J, Regli F. Iscemic stroke in adults younger than 30 years of age: Cause and prognosis. Arch Neurol 1987;44:479-82

16. BhowmikNB, Abbas A, Saifuddin M,IslamR, Habib R,RahmanA, et al. Ischemic Strokes: Observations from a Hospital Based StrokeRegistry in Bangladesh.Hindawi Publishing Corporation Stroke Research and Treatment 2016; Article ID 5610797:13.

17. Tatu L, Moulin T, Bogousslavsky J, Duvernoy H. Arterial territories of human brain: brainstem and cerebellum. Neurology 1996; 47:1125-35.

18. Barlas NY, Putaala J, Waje-Andreassen U. Etiology of firstever ischaemic stroke in European young adults: the 15 cities young stroke study. Eur J Neurol 2013;20:1431-39.

19. Tatu L, Moulin T, Bogousslavsky J, Duvernoy H. Arterial territories of the human brain: cerebral hemispheres. Neurology 1998;50:1699-1708.

20. Nedeltchev K, der Maur TA, Georgiadis D, Arnold M, Caso $\mathrm{V}$, Mattle HP, et al. Ischemic stroke in young adults: predictors of outcome and recurrence. J Neurol Neurosurg Psychiatry 2005;76:191-95. 